CRYSTALLOGRAPHIC COMMUNICATIONS

ISSN 2056-9890

Received 21 November 2014

Accepted 1 December 2014

Edited by $\mathrm{H}$. Stoeckli-Evans, University of Neuchâtel, Switzerland

Keywords: crystal structure; Schiff base; azines; dimers; $\mathrm{C}-\mathrm{H} \cdots \pi$ contacts

CCDC reference: 1036846

Supporting information: this article has supporting information at journals.iucr.org/e

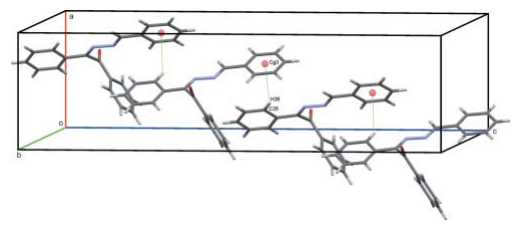

OPEN $\odot$ ACCESS

\section{Crystal structure of $(Z)-2-[(E)$-2-benzylidene- hydrazin-1-ylidene]-1,2-diphenylethanone}

\author{
Abdelaziz Bouchama, ${ }^{a}$ Messaoud Yahiaoui, ${ }^{\text {b }}$ Chaabane Chiter, ${ }^{\mathrm{b}}$ Zouaoui Setifi ${ }^{\mathrm{c}, \mathrm{a} *}$ \\ and Jim Simpson ${ }^{d}$
}

\begin{abstract}
aLaboratoire de Chimie, Ingénierie Moléculaire et Nanostructures (LCIMN), Université Ferhat Abbas Sétif 1, Sétif 19000, Algeria, 'baboratoire d'Electrochimie des Matériaux Moléculaires et Complexes (L.E.M.M.C.), Université Ferhat Abbas Sétif 1, Sétif 19000, Algeria, 'Département de Technologie, Faculté de Technologie, Université 20 Août 1955-Skikda, BP 26, Route d'El-Hadaiek, Skikda 21000, Algeria, and 'Department of Chemistry, University of Otago, PO Box 56, Dunedin, New Zealand. *Correspondence e-mail: setifi_zouaoui@yahoo.fr
\end{abstract}

The title compound, $\mathrm{C}_{21} \mathrm{H}_{16} \mathrm{~N}_{2} \mathrm{O}$, has an almost planar (r.m.s. deviation = $0.0074 \AA$ ) 1,2-dibenzylidenehydrazine backbone with an approximately orthogonal almost planar (r.m.s. deviation $=0.0368 \AA$ ) phenylethanone substituent on one of the imine $\mathrm{C}$ atoms. The dihedral angle between the two mean planes is $76.99(4)^{\circ}$. In the crystal, molecules are linked via $\mathrm{C}-\mathrm{H} \cdots \mathrm{O}$ hydrogen bonds and $\mathrm{C}-\mathrm{H} \cdots \pi$ contacts, forming a three-dimensional structure with molecules stacked along the $a$-axis direction.

\section{Chemical context}

Aromatic carbonyl compounds react easily with hydrazines to form hydrazones, which can condense with a second molecule of a carbonyl compound to yield an azine. As a result of their fascinating physical and chemical properties, azines and their derivatives have been utilized extensively in areas such as dyes (Kim et al., 2010) and non-linear fluorophores (Facchetti et al., 2002). They are also noted for their biological and pharmaceutical applications (Wadher et al., 2009; Pandeya et al., 1999). Furthermore, there are many reports of polyazines as highly conjugated polymers functioning in electronic, optoelectronic and photonic applications (Dudis et al., 1993). As part of our studies of Schiff base azines, the title compound was synthesized and its molecular and crystal structure are reported on herein.

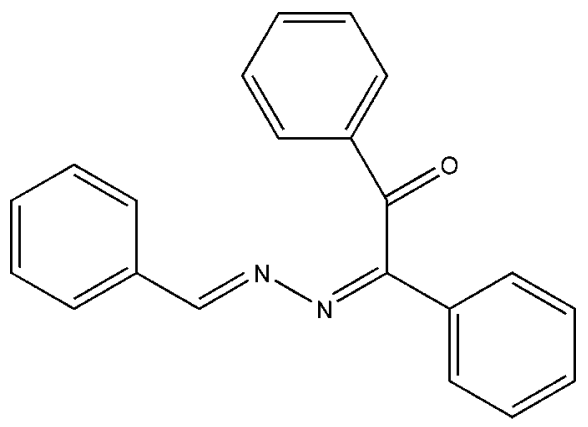

\section{Structural commentary}

The molecule of the title compound, Fig. 1, comprises a 1,2dibenzylidenehydrazine backbone with a phenyl ethanone substituent on atom $\mathrm{C} 2$. Both the hydrazine and ethanone fragments are approximately planar with r.m.s. deviations of $0.0074 \AA$ from the $\mathrm{O} 1 / \mathrm{C} 1 / \mathrm{C} 11-\mathrm{C} 16$ mean plane and $0.0368 \AA$ from the plane through the 16 atoms of the dibenzyl- 


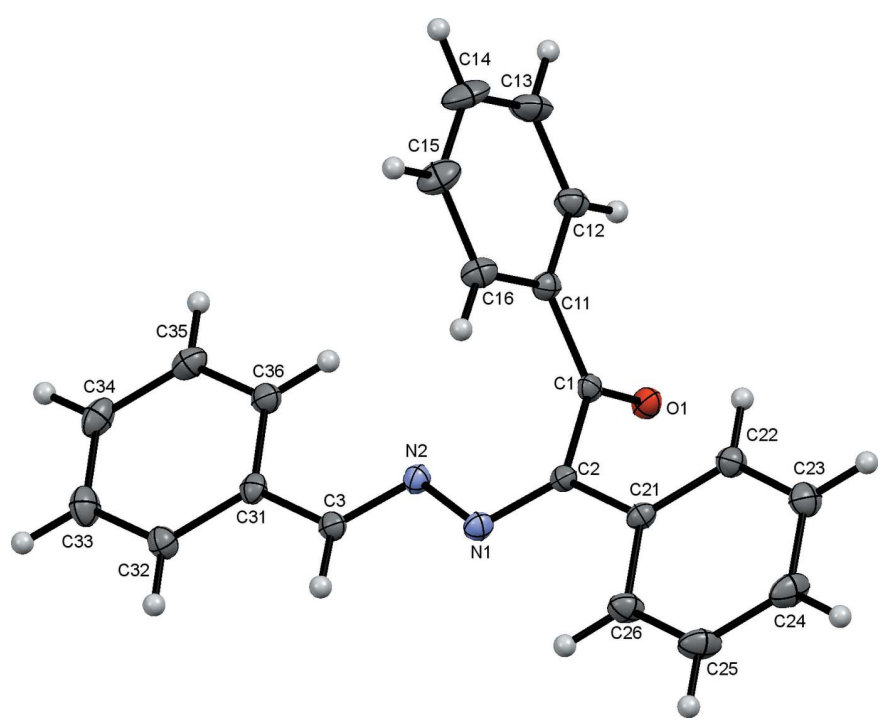

Figure 1

The molecular structure of the title compound, showing the atom labelling. Displacement ellipsoids are drawn at the $50 \%$ probability level.

idenehydrazine unit. The two mean planes are almost orthogonal with a dihedral angle of $76.99(4)^{\circ}$. The molecule adopts a $Z$ conformation with respect to the $\mathrm{C} 2=\mathrm{N} 1$ bond and an $E$ conformation with respect to the $\mathrm{C} 3=\mathrm{N} 2$ bond, with the carbonyl atom $\mathrm{O} 1$ and the $\mathrm{C} 11-\mathrm{C} 16$ phenyl ring located on opposite sides of the dibenzylidenehydrazine plane. The bond lengths and angles in the title molecule agree reasonably well with those found in closely related structures (Abbasi et al., 2007; Wieland et al., 2011).

\section{Supramolecular features}

In the crystal, a pair of $\mathrm{C} 35-\mathrm{H} 35 \cdots \mathrm{O} 1$ hydrogen bonds link adjacent molecules into dimers with $R_{2}^{2}(20)$ ring motifs (Fig. 2 and Table 1). Atom $\mathrm{O} 1$ is also involved in two further $\mathrm{C}-$

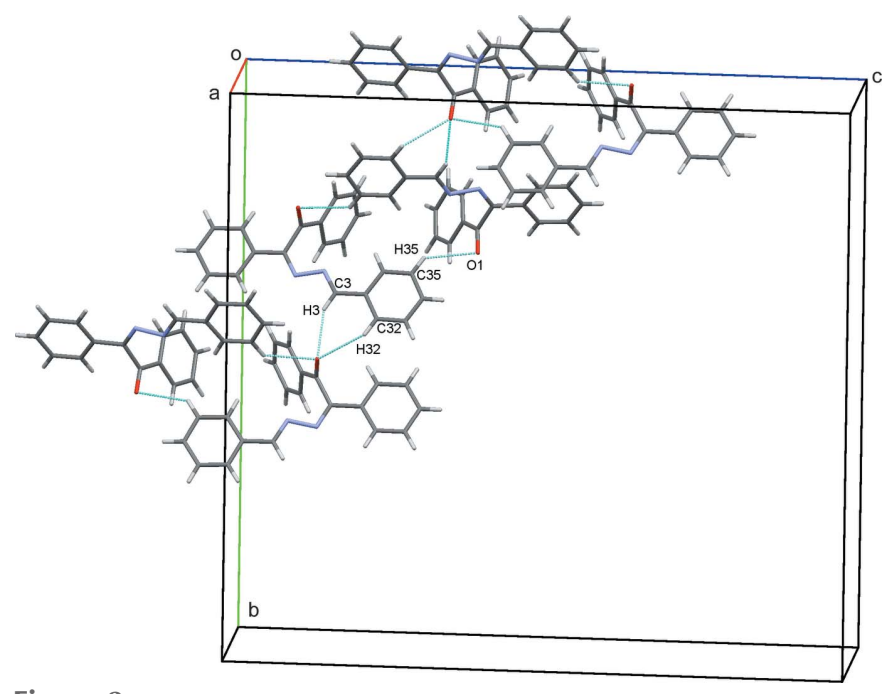

Figure 2

A view of the dimers formed via $\mathrm{C}-\mathrm{H} \cdots \mathrm{O}$ contacts (blue dashed lines; see Table 1 for details) and linked into stacks running parallel to (011) in the crystal of the title compound.
Table 1

Hydrogen-bond geometry $\left(\AA,^{\circ}\right)$.

$\mathrm{Cg}$ is the centroid of the $\mathrm{C} 31-\mathrm{C} 36$ phenyl ring.

\begin{tabular}{lllll}
\hline$D-\mathrm{H} \cdots A$ & $D-\mathrm{H}$ & $\mathrm{H} \cdots A$ & $D \cdots A$ & $D-\mathrm{H} \cdots A$ \\
\hline $\mathrm{C} 35-\mathrm{H} 35 \cdots \mathrm{O} 1^{\mathrm{i}}$ & 0.95 & 2.61 & $3.337(3)$ & 134 \\
$\mathrm{C} 3-\mathrm{H} 3 \cdots \mathrm{O} 1^{\mathrm{ii}}$ & 0.95 & 2.41 & $3.272(3)$ & 151 \\
$\mathrm{C} 32-\mathrm{H} 32 \cdots \mathrm{O} 1^{\mathrm{ii}}$ & 0.95 & 2.68 & $3.478(3)$ & 141 \\
$\mathrm{C} 26-\mathrm{H} 26 \cdots \mathrm{gg}^{\mathrm{iii}}$ & 0.95 & 2.97 & $3.699(3)$ & 135 \\
\hline
\end{tabular}

Symmetry codes: (i) $x,-y+\frac{1}{2},-z+\frac{1}{2}$; (ii) $x+\frac{1}{4}, y-\frac{1}{4},-z+\frac{3}{4}$; (iii) $x+\frac{1}{4},-y+\frac{1}{4}, z+\frac{1}{4}$.

$\mathrm{H} \cdots \mathrm{O}$ hydrogen bonds, $\mathrm{C} 3-\mathrm{H} 3 \cdots \mathrm{O} 1$ and $\mathrm{C} 32-\mathrm{H} 32 \cdots \mathrm{O} 1$ that generate $R_{2}^{1}(6)$ ring motifs. These contacts link the dimers into stacks parallel to (011); see Table 1 and Fig. 2. Interestingly, neither of the hydrazine $\mathrm{N}$ atoms are involved in significantly close intermolecular contacts with the shortest intermolecular $\mathrm{H} 12 \cdots \mathrm{N} 1$ contact being $c a 2.85 \AA$. A contribution to the packing is, however, made by a $\mathrm{C}-\mathrm{H} \cdots \pi$

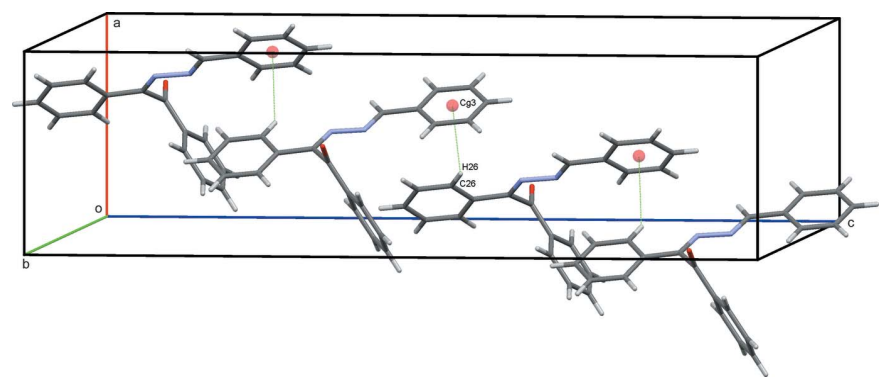

Figure 3

A view of the chains along the $c$-axis direction formed by $\mathrm{C}-\mathrm{H} \cdots \pi$ contacts in the crystal of the title compound (shown as green dotted lines with the ring centroids displayed as coloured spheres, see Table 1 for details).

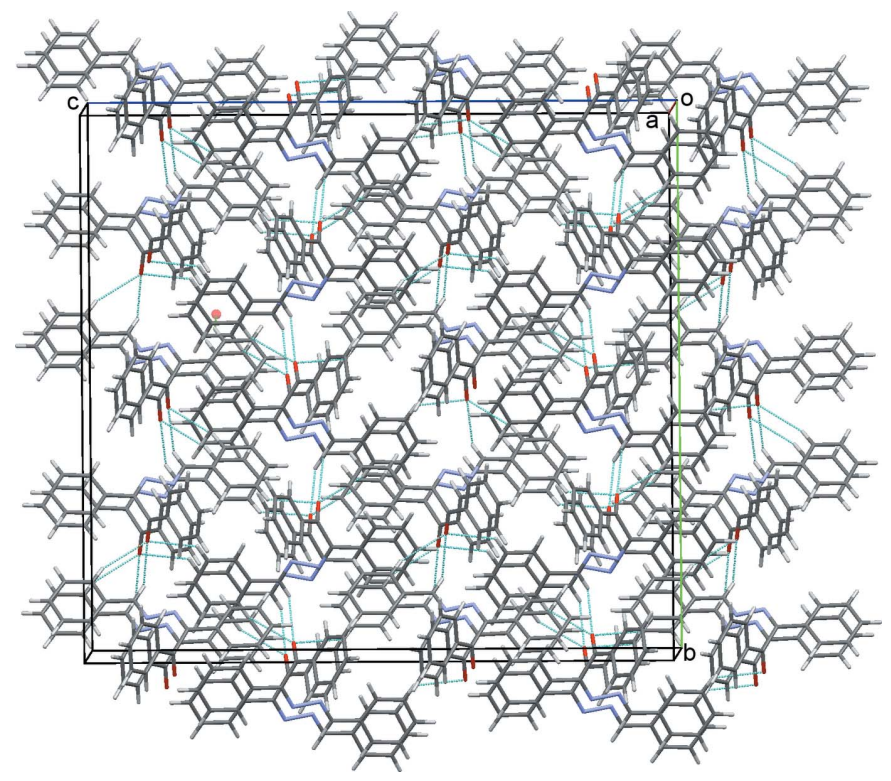

Figure 4

A view along the $a$-axis direction of the crystal packing of the title compound. Hydrogen bonds are drawn as blue dashed lines with a representative $\mathrm{C}-\mathrm{H} \cdots \pi$ contact shown as a green dotted line (see Table 1 for details). 
Table 2

Experimental details.

\begin{tabular}{|c|c|}
\hline \multicolumn{2}{|l|}{ Crystal data } \\
\hline Chemical formula & $\mathrm{C}_{21} \mathrm{H}_{16} \mathrm{~N}_{2} \mathrm{O}$ \\
\hline$M_{\mathrm{r}}$ & 312.36 \\
\hline Crystal system, space group & Orthorhombic, $F 2 d d$ \\
\hline Temperature $(\mathrm{K})$ & 150 \\
\hline$a, b, c(\AA)$ & $\begin{array}{l}8.1653(3), 27.6113(11), \\
29.6818(13)\end{array}$ \\
\hline$V\left(\AA^{3}\right)$ & $6691.9(5)$ \\
\hline$Z$ & 16 \\
\hline Radiation type & Мо $K \alpha$ \\
\hline$\mu\left(\mathrm{mm}^{-1}\right)$ & 0.08 \\
\hline Crystal size $(\mathrm{mm})$ & $0.55 \times 0.29 \times 0.24$ \\
\hline \multicolumn{2}{|l|}{ Data collection } \\
\hline Diffractometer & Bruker APEXII \\
\hline Absorption correction & $\begin{array}{l}\text { Multi-scan (SADABS; Bruker, } \\
\text { 2006) }\end{array}$ \\
\hline$T_{\min }, T_{\max }$ & $0.884,0.982$ \\
\hline $\begin{array}{l}\text { No. of measured, independent and } \\
\text { observed }[I>2 \sigma(I)] \text { reflections }\end{array}$ & $8049,3350,3036$ \\
\hline$R_{\text {int }}$ & 0.032 \\
\hline$(\sin \theta / \lambda)_{\max }\left(\AA^{-1}\right)$ & 0.649 \\
\hline \multicolumn{2}{|l|}{ Refinement } \\
\hline$R\left[F^{2}>2 \sigma\left(F^{2}\right)\right], w R\left(F^{2}\right), S$ & $0.039,0.094,1.06$ \\
\hline No. of reflections & 3350 \\
\hline No. of parameters & 217 \\
\hline No. of restraints & 1 \\
\hline $\mathrm{H}$-atom treatment & $\mathrm{H}$-atom parameters constrained \\
\hline$\Delta \rho_{\max }, \Delta \rho_{\min }\left(\mathrm{e} \AA^{-3}\right)$ & $0.18,-0.16$ \\
\hline
\end{tabular}

Computer programs: APEX2 and SAINT (Bruker, 2006), SIR97 (Altomare et al., 1999), SHELXL2014 (Sheldrick, 2008), Mercury (Macrae et al., 2008), CRYSCAL (T. Roisnel, local program), enCIFer (Allen et al., 2004), PLATON (Spek, 2009), WinGX (Farrugia, 2012) and publCIF (Westrip 2010).

interaction (Table 1). These interactions link molecules in a head-to-tail fashion, forming chains along $c$, as shown in Fig. 3. With 16 molecules in the orthorhombic unit cell, these various contacts combine to form a three dimensional structure with molecules stacked along the $a$-axis direction, as shown in Fig. 4 .

\section{Database survey}

A search for the (benzylidenehydrazono)-1,2-diphenylethanone skeleton in the Cambridge Structural Database (Version 5.35, November 2013 with three updates; Groom \& Allen, 2014) revealed only 7 similar compounds. The closest to the title structure are 2-\{(Z)-2-[(E)-1-(2-hydroxyphenyl)methylidene]hydrazono\}-1,2-diphenylethan-1-one (Abbasi et $a l ., 2007)$, with an hydroxy substituent in the $p$ position on the equivalent of the benzene ring, and 1,2-diphenyl-2-[4-(4pyridyl)benzylidenehydrazono]ethan-1-one, with a pyridyl ring in the same position (Patra \& $\mathrm{Ng}, 2009$ ). Two reports of polymorphs of the symmetrical 2,2'-(1,2-hydrazinediylidene)bis(diphenylethanone) have also appeared (Patra et al., 2009; Wieland et al., 2011)

\section{Synthesis and crystallization}

A mixture of benzaldehyde $(0.01 \mathrm{~mol}, 1.06 \mathrm{~g})$, benzil $(0.01 \mathrm{~mol}, 2.10 \mathrm{~g})$ and hydrazine hydrate $(0.01 \mathrm{~mol}, 0.32 \mathrm{~g})$ in $50 \mathrm{ml}$ of ethanol containing 2 drops of acetic acid was refluxed for about $2 \mathrm{~h}$. The reaction was monitored by TLC until completion. Excess solvent was evaporated under vacuum and the resulting yellow solid product was recrystallized from absolute ethanol to afford yellow needles of the title compound (m.p. $453 \mathrm{~K}, 75 \%$ yield). Analysis calculated for $\mathrm{C}_{21} \mathrm{H}_{16} \mathrm{~N}_{2} \mathrm{O}$ (312.36): C 80.75, H 5.16, N 8.97\%; found: C 80.73, H 5.17, N 9.01\%.

\section{Refinement}

Crystal data, data collection and structure refinement details are summarized in Table 2. The $\mathrm{C}$-bound $\mathrm{H}$ atoms were included in calculated positions and treated as riding atoms: $\mathrm{C}-\mathrm{H}=0.95 \AA$ with $U_{\text {iso }}=1.2 U_{\text {eq }}(\mathrm{C})$.

\section{Acknowledgements}

The authors acknowledge the Algerian Ministry of Higher Education and Scientific Research, the Algerian Directorate General for Scientific Research and Technological Development, and Ferhat Abbas Sétif 1 University for financial support. The Chemistry Department of the University of Otago is also thanked for support of the work of JS. Dr Lahcène Ouahab from the University of Rennes 1, France, is thanked for the data collection.

\section{References}

Abbasi, A., Mohammadi Ziarani, G. \& Tarighi, S. (2007). Acta Cryst. E63, o2579-o2580.

Allen, F. H., Johnson, O., Shields, G. P., Smith, B. R. \& Towler, M. (2004). J. Appl. Cryst. 37, 335-338.

Altomare, A., Burla, M. C., Camalli, M., Cascarano, G. L., Giacovazzo, C., Guagliardi, A., Moliterni, A. G. G., Polidori, G. \& Spagna, R. (1999). J. Appl. Cryst. 32, 115-119.

Bruker (2006). APEX2, SAINT and SADABS. Bruker AXS Inc., Madison, Wisconsin, USA.

Dudis, D. S., Yeates, A. T., Kost, D., Smith, D. A. \& Medrano, J. (1993). J. Am. Chem. Soc. 115, 8770-8774.

Facchetti, A., Abbotto, A., Beverina, L., van der Boom, M. E., Dutta, P., Evmenenko, G., Marks, T. J. \& Pagani, G. A. (2002). Chem. Mater. 14, 4996-5005.

Farrugia, L. J. (2012). J. Appl. Cryst. 45, 849-854.

Groom, C. R. \& Allen, F. H. (2014). Angew. Chem. Int. Ed. 53, 662671.

Kim, S. H., Gwon, S. Y., Burkinshaw, S. M. \& Son, Y. A. (2010). Dyes Pigm. 87, 268-271.

Macrae, C. F., Bruno, I. J., Chisholm, J. A., Edgington, P. R., McCabe, P., Pidcock, E., Rodriguez-Monge, L., Taylor, R., van de Streek, J. \& Wood, P. A. (2008). J. Appl. Cryst. 41, 466-470.

Pandeya, S. N., Sriram, D., Nath, G. \& De Clercq, E. (1999). Pharm. Acta Helv. 74, 11-17.

Patra, G. K., Mukherjee, A. \& Ng, S. W. (2009). Acta Cryst. E65, 01745.

Patra, G. K. \& Ng, S. W. (2009). Acta Cryst. E65, o1810.

Sheldrick, G. M. (2008). Acta Cryst. A64, 112-122.

Spek, A. L. (2009). Acta Cryst. D65, 148-155.

Wadher, J. S., Puranik, M. P., Karande, N. A. \& Yeole, P. G. (2009). J. Pharm. Tech. Res. 1, 22-33.

Westrip, S. P. (2010). J. Appl. Cryst. 43, 920-925.

Wieland, M., Seichter, W., Schwarzer, A. \& Weber, E. (2011). Struct. Chem. 22, 1267-1279. 


\section{supporting information}

Acta Cryst. (2015). E71, 35-37 [https://doi.org/10.1107/S2056989014026358]

Crystal structure of (Z)-2-[(E)-2-benzylidenehydrazin-1-ylidene]-1,2-diphenylethanone

Abdelaziz Bouchama, Messaoud Yahiaoui, Chaabane Chiter, Zouaoui Setifi and Jim Simpson

Computing details

Data collection: APEX2 (Bruker, 2006); cell refinement: SAINT (Bruker, 2006); data reduction: SAINT (Bruker, 2006); program(s) used to solve structure: SIR97 (Altomare et al., 1999); program(s) used to refine structure: SHELXL2014 (Sheldrick, 2008); molecular graphics: Mercury (Macrae et al., 2008); software used to prepare material for publication: CRYSCAL (T. Roisnel, local program), SHELXL2014 (Sheldrick, 2008), enCIFer (Allen et al., 2004), PLATON (Spek, 2009), publCIF (Westrip 2010) and WinGX (Farrugia, 2012).

(Z)-2-[(E)-2-Benzylidenehydrazin-1-ylidene]-1,2-diphenylethanone

Crystal data

$\mathrm{C}_{21} \mathrm{H}_{16} \mathrm{~N}_{2} \mathrm{O}$

$M_{r}=312.36$

Orthorhombic, F2dd

Hall symbol: F $-2 \mathrm{~d} 2$

$a=8.1653(3) \AA$

$b=27.6113(11) \AA$

$c=29.6818(13) \AA$

$V=6691.9(5) \AA^{3}$

$Z=16$

Data collection

Bruker APEXII diffractometer

Radiation source: fine-focus sealed tube CCD rotation images, thin slices scans Absorption correction: multi-scan

(SADABS; Bruker, 2006)

$T_{\text {min }}=0.884, T_{\max }=0.982$

8049 measured reflections

\section{Refinement}

Refinement on $F^{2}$

Least-squares matrix: full

$R\left[F^{2}>2 \sigma\left(F^{2}\right)\right]=0.039$

$w R\left(F^{2}\right)=0.094$

$S=1.06$

3350 reflections

217 parameters

1 restraint
$F(000)=2624$

$D_{\mathrm{x}}=1.240 \mathrm{Mg} \mathrm{m}^{-3}$

Mo $K \alpha$ radiation, $\lambda=0.71073 \AA$

Cell parameters from 2807 reflections

$\theta=2.7-27.3^{\circ}$

$\mu=0.08 \mathrm{~mm}^{-1}$

$T=150 \mathrm{~K}$

Prism, yellow

$0.55 \times 0.29 \times 0.24 \mathrm{~mm}$

3350 independent reflections

3036 reflections with $I>2 \sigma(I)$

$R_{\text {int }}=0.032$

$\theta_{\max }=27.5^{\circ}, \theta_{\min }=3.0^{\circ}$

$h=-9 \rightarrow 10$

$k=-35 \rightarrow 24$

$l=-38 \rightarrow 38$

Hydrogen site location: inferred from neighbouring sites

$\mathrm{H}$-atom parameters constrained

$w=1 /\left[\sigma^{2}\left(F_{\mathrm{o}}^{2}\right)+(0.0407 P)^{2}+4.1058 P\right]$

where $P=\left(F_{\mathrm{o}}{ }^{2}+2 F_{\mathrm{c}}{ }^{2}\right) / 3$

$(\Delta / \sigma)_{\max }<0.001$

$\Delta \rho_{\max }=0.18$ e $\AA^{-3}$

$\Delta \rho_{\min }=-0.16 \mathrm{e} \AA^{-3}$ 


\section{Special details}

Geometry. All e.s.d.'s (except the e.s.d. in the dihedral angle between two 1.s. planes) are estimated using the full covariance matrix. The cell e.s.d.'s are taken into account individually in the estimation of e.s.d.'s in distances, angles and torsion angles; correlations between e.s.d.'s in cell parameters are only used when they are defined by crystal symmetry. An approximate (isotropic) treatment of cell e.s.d.'s is used for estimating e.s.d.'s involving l.s. planes.

Fractional atomic coordinates and isotropic or equivalent isotropic displacement parameters $\left(\AA^{2}\right)$

\begin{tabular}{|c|c|c|c|c|}
\hline & $x$ & $y$ & $z$ & $U_{\text {iso }} * / U_{\text {eq }}$ \\
\hline $\mathrm{C} 11$ & $0.4692(3)$ & $0.24563(8)$ & $0.36467(7)$ & $0.0255(5)$ \\
\hline $\mathrm{C} 12$ & $0.4156(3)$ & $0.28709(9)$ & $0.34210(7)$ & $0.0316(5)$ \\
\hline H12 & 0.4758 & 0.3164 & 0.3444 & $0.038^{*}$ \\
\hline $\mathrm{C} 13$ & $0.2746(3)$ & $0.28525(11)$ & $0.31643(9)$ & $0.0426(7)$ \\
\hline H13 & 0.2381 & 0.3134 & 0.3010 & $0.051 *$ \\
\hline $\mathrm{C} 14$ & $0.1865(3)$ & $0.24267(12)$ & $0.31313(10)$ & $0.0504(8)$ \\
\hline H14 & 0.0888 & 0.2418 & 0.2958 & $0.060^{*}$ \\
\hline $\mathrm{C} 15$ & 0.2393 & $0.20147(11)$ & $0.33483(10)$ & $0.0453(7)$ \\
\hline H15 & 0.1790 & 0.1722 & 0.3322 & $0.054^{*}$ \\
\hline $\mathrm{C} 16$ & $0.3810(3)$ & $0.20282(9)$ & $0.36059(8)$ & $0.0326(6)$ \\
\hline H16 & 0.4178 & 0.1744 & 0.3755 & $0.039^{*}$ \\
\hline $\mathrm{C} 1$ & $0.6212(3)$ & $0.24786(8)$ & $0.39198(6)$ & $0.0222(4)$ \\
\hline O1 & $0.7037(2)$ & $0.28433(5)$ & $0.39542(5)$ & $0.0300(4)$ \\
\hline $\mathrm{C} 2$ & $0.6774(3)$ & $0.20274(8)$ & $0.41744(7)$ & $0.0220(5)$ \\
\hline $\mathrm{C} 21$ & $0.6542(3)$ & $0.20065(8)$ & $0.46670(6)$ & $0.0234(5)$ \\
\hline $\mathrm{C} 22$ & $0.5622(3)$ & $0.23566(9)$ & $0.48904(7)$ & $0.0291(5)$ \\
\hline $\mathrm{H} 22$ & 0.5110 & 0.2608 & 0.4723 & $0.035^{*}$ \\
\hline $\mathrm{C} 23$ & $0.5449(3)$ & $0.23409(10)$ & $0.53561(8)$ & $0.0344(6)$ \\
\hline $\mathrm{H} 23$ & 0.4825 & 0.2582 & 0.5507 & $0.041^{*}$ \\
\hline $\mathrm{C} 24$ & $0.6182(3)$ & $0.19755(11)$ & $0.55991(8)$ & $0.0401(6)$ \\
\hline $\mathrm{H} 24$ & 0.6067 & 0.1966 & 0.5917 & $0.048^{*}$ \\
\hline $\mathrm{C} 25$ & $0.7083(4)$ & $0.16228(11)$ & $0.53816(8)$ & $0.0406(7)$ \\
\hline $\mathrm{H} 25$ & 0.7575 & 0.1369 & 0.5550 & $0.049^{*}$ \\
\hline $\mathrm{C} 26$ & $0.7272(3)$ & $0.16376(9)$ & $0.49169(8)$ & $0.0332(6)$ \\
\hline $\mathrm{H} 26$ & 0.7901 & 0.1396 & 0.4769 & $0.040^{*}$ \\
\hline N1 & $0.7555(2)$ & $0.16872(7)$ & $0.39690(6)$ & $0.0265(4)$ \\
\hline N2 & $0.7675(2)$ & $0.17867(7)$ & $0.35026(6)$ & $0.0255(4)$ \\
\hline $\mathrm{C} 3$ & $0.8450(3)$ & $0.14520(8)$ & $0.32965(7)$ & $0.0245(5)$ \\
\hline H3 & 0.8903 & 0.1192 & 0.3465 & $0.029^{*}$ \\
\hline C31 & $0.8657(3)$ & $0.14596(8)$ & $0.28078(7)$ & $0.0252(5)$ \\
\hline $\mathrm{C} 32$ & $0.9510(3)$ & $0.10863(9)$ & $0.25997(8)$ & $0.0307(5)$ \\
\hline $\mathrm{H} 32$ & 0.9991 & 0.0838 & 0.2777 & $0.037^{*}$ \\
\hline C33 & $0.9664(3)$ & $0.10743(10)$ & $0.21338(8)$ & $0.0383(6)$ \\
\hline H33 & 1.0228 & 0.0814 & 0.1993 & $0.046^{*}$ \\
\hline C34 & $0.9002(3)$ & $0.14389(9)$ & $0.18749(8)$ & $0.0371(6)$ \\
\hline H34 & 0.9115 & 0.1431 & 0.1556 & $0.045^{*}$ \\
\hline $\mathrm{C} 35$ & $0.8166(3)$ & $0.18203(10)$ & $0.20799(8)$ & $0.0346(6)$ \\
\hline H35 & 0.7721 & 0.2074 & 0.1902 & $0.041 *$ \\
\hline $\mathrm{C} 36$ & $0.7987(3)$ & $0.18291(9)$ & $0.25427(8)$ & $0.0294(5)$ \\
\hline
\end{tabular}


Atomic displacement parameters $\left(\AA^{2}\right)$

\begin{tabular}{lllllll}
\hline & $U^{11}$ & $U^{22}$ & $U^{33}$ & $U^{12}$ & $U^{13}$ & $U^{23}$ \\
\hline C11 & $0.0250(11)$ & $0.0314(12)$ & $0.0200(9)$ & $0.0026(11)$ & $0.0015(8)$ & $0.0014(8)$ \\
C12 & $0.0328(13)$ & $0.0337(13)$ & $0.0283(11)$ & $0.0066(11)$ & $-0.0003(10)$ & $0.0044(10)$ \\
C13 & $0.0368(15)$ & $0.0525(17)$ & $0.0385(14)$ & $0.0136(14)$ & $-0.0061(11)$ & $0.0126(12)$ \\
C14 & $0.0283(15)$ & $0.076(2)$ & $0.0472(15)$ & $-0.0018(15)$ & $-0.0157(12)$ & $0.0105(15)$ \\
C15 & $0.0348(15)$ & $0.0542(17)$ & $0.0471(15)$ & $-0.0116(14)$ & $-0.0096(12)$ & $0.0049(13)$ \\
C16 & $0.0298(13)$ & $0.0375(14)$ & $0.0304(11)$ & $-0.0016(12)$ & $-0.0019(9)$ & $0.0049(10)$ \\
C1 & $0.0268(11)$ & $0.0233(11)$ & $0.0166(8)$ & $0.0035(10)$ & $0.0021(8)$ & $-0.0012(8)$ \\
O1 & $0.0370(10)$ & $0.0233(8)$ & $0.0298(8)$ & $-0.0021(8)$ & $-0.0063(7)$ & $0.0013(6)$ \\
C2 & $0.0214(11)$ & $0.0228(10)$ & $0.0217(9)$ & $-0.0008(10)$ & $-0.0023(8)$ & $0.0004(8)$ \\
C21 & $0.0236(12)$ & $0.0262(11)$ & $0.0205(9)$ & $-0.0027(9)$ & $-0.0012(8)$ & $0.0029(8)$ \\
C22 & $0.0292(13)$ & $0.0311(13)$ & $0.0268(11)$ & $0.0028(11)$ & $0.0008(9)$ & $0.0009(9)$ \\
C23 & $0.0317(14)$ & $0.0428(15)$ & $0.0288(12)$ & $0.0041(12)$ & $0.0054(10)$ & $-0.0010(10)$ \\
C24 & $0.0344(14)$ & $0.0655(18)$ & $0.0203(10)$ & $0.0006(14)$ & $0.0028(10)$ & $0.0063(12)$ \\
C25 & $0.0372(15)$ & $0.0549(17)$ & $0.0297(12)$ & $0.0075(14)$ & $-0.0009(10)$ & $0.0153(11)$ \\
C26 & $0.0347(14)$ & $0.0367(14)$ & $0.0284(12)$ & $0.0061(12)$ & $0.0004(10)$ & $0.0079(10)$ \\
N1 & $0.0320(11)$ & $0.0263(10)$ & $0.0212(9)$ & $0.0019(9)$ & $-0.0025(8)$ & $0.0019(7)$ \\
N2 & $0.0317(11)$ & $0.0248(10)$ & $0.0201(9)$ & $0.0012(9)$ & $-0.0014(8)$ & $-0.0013(7)$ \\
C3 & $0.0258(12)$ & $0.0213(11)$ & $0.0265(10)$ & $-0.0013(10)$ & $-0.0021(8)$ & $0.0005(9)$ \\
C31 & $0.0237(12)$ & $0.0250(11)$ & $0.0268(10)$ & $-0.0049(10)$ & $-0.0009(9)$ & $-0.0034(9)$ \\
C32 & $0.0327(14)$ & $0.0296(12)$ & $0.0299(12)$ & $0.0014(11)$ & $0.0035(9)$ & $-0.0009(9)$ \\
C33 & $0.0395(16)$ & $0.0419(15)$ & $0.0335(13)$ & $-0.0008(13)$ & $0.0100(10)$ & $-0.0070(11)$ \\
C34 & $0.0386(15)$ & $0.0490(16)$ & $0.0238(10)$ & $-0.0104(13)$ & $0.0031(10)$ & $-0.0019(10)$ \\
C35 & $0.0359(14)$ & $0.0378(14)$ & $0.0299(12)$ & $-0.0038(12)$ & $-0.0064(10)$ & $0.0054(10)$ \\
C36 & $0.0319(13)$ & $0.0256(12)$ & $0.0307(11)$ & $-0.0002(11)$ & $-0.0049(10)$ & $0.0005(9)$ \\
& & & & & &
\end{tabular}

Geometric parameters $\left(\AA,{ }^{\circ}\right)$

\begin{tabular}{llll}
\hline $\mathrm{C} 11-\mathrm{C} 16$ & $1.390(3)$ & $\mathrm{C} 23-\mathrm{H} 23$ & 0.9500 \\
$\mathrm{C} 11-\mathrm{C} 12$ & $1.397(3)$ & $\mathrm{C} 24-\mathrm{C} 25$ & $1.381(4)$ \\
$\mathrm{C} 11-\mathrm{C} 1$ & $1.483(3)$ & $\mathrm{C} 24-\mathrm{H} 24$ & 0.9500 \\
$\mathrm{C} 12-\mathrm{C} 13$ & $1.382(4)$ & $\mathrm{C} 25-\mathrm{C} 26$ & $1.388(3)$ \\
$\mathrm{C} 12-\mathrm{H} 12$ & 0.9500 & $\mathrm{C} 25-\mathrm{H} 25$ & 0.9500 \\
$\mathrm{C} 13-\mathrm{C} 14$ & $1.382(4)$ & $\mathrm{C} 26-\mathrm{H} 26$ & 0.9500 \\
$\mathrm{C} 13-\mathrm{H} 13$ & 0.9500 & $\mathrm{~N} 1-\mathrm{N} 2$ & $1.415(2)$ \\
$\mathrm{C} 14-\mathrm{C} 15$ & $1.377(4)$ & $\mathrm{N} 2-\mathrm{C} 3$ & $1.276(3)$ \\
$\mathrm{C} 14-\mathrm{H} 14$ & 0.9500 & $\mathrm{C} 3-\mathrm{C} 31$ & $1.461(3)$ \\
$\mathrm{C} 15-\mathrm{C} 16$ & $1.387(4)$ & $\mathrm{C} 3-\mathrm{H} 3$ & 0.9500 \\
$\mathrm{C} 15-\mathrm{H} 15$ & 0.9500 & $\mathrm{C} 31-\mathrm{C} 32$ & $1.389(3)$ \\
$\mathrm{C} 16-\mathrm{H} 16$ & 0.9500 & $\mathrm{C} 31-\mathrm{C} 36$ & $1.400(3)$ \\
$\mathrm{C} 1-\mathrm{O} 1$ & $1.216(3)$ & $\mathrm{C} 32-\mathrm{C} 33$ & $1.389(3)$ \\
$\mathrm{C} 1-\mathrm{C} 2$ & $1.528(3)$ & $\mathrm{C} 32-\mathrm{H} 32$ & 0.9500 \\
$\mathrm{C} 2-\mathrm{N} 1$ & $1.288(3)$ & $\mathrm{C} 33-\mathrm{C} 34$ & $1.377(4)$ \\
$\mathrm{C} 2-\mathrm{C} 21$ & $1.476(3)$ & $\mathrm{C} 33-\mathrm{H} 33$ & 0.9500
\end{tabular}




\begin{tabular}{|c|c|c|c|}
\hline $\mathrm{C} 21-\mathrm{C} 22$ & $1.392(3)$ & $\mathrm{C} 34-\mathrm{C} 35$ & $1.395(4)$ \\
\hline $\mathrm{C} 21-\mathrm{C} 26$ & $1.394(3)$ & $\mathrm{C} 34-\mathrm{H} 34$ & 0.9500 \\
\hline $\mathrm{C} 22-\mathrm{C} 23$ & $1.390(3)$ & $\mathrm{C} 35-\mathrm{C} 36$ & $1.382(3)$ \\
\hline $\mathrm{C} 22-\mathrm{H} 22$ & 0.9500 & $\mathrm{C} 35-\mathrm{H} 35$ & 0.9500 \\
\hline $\mathrm{C} 23-\mathrm{C} 24$ & $1.377(4)$ & $\mathrm{C} 36-\mathrm{H} 36$ & 0.9500 \\
\hline $\mathrm{C} 16-\mathrm{C} 11-\mathrm{C} 12$ & $119.5(2)$ & $\mathrm{C} 23-\mathrm{C} 24-\mathrm{C} 25$ & $120.2(2)$ \\
\hline $\mathrm{C} 16-\mathrm{C} 11-\mathrm{C} 1$ & $121.1(2)$ & $\mathrm{C} 23-\mathrm{C} 24-\mathrm{H} 24$ & 119.9 \\
\hline $\mathrm{C} 12-\mathrm{C} 11-\mathrm{C} 1$ & $119.4(2)$ & $\mathrm{C} 25-\mathrm{C} 24-\mathrm{H} 24$ & 119.9 \\
\hline $\mathrm{C} 13-\mathrm{C} 12-\mathrm{C} 11$ & $119.7(2)$ & $\mathrm{C} 24-\mathrm{C} 25-\mathrm{C} 26$ & $120.2(2)$ \\
\hline $\mathrm{C} 13-\mathrm{C} 12-\mathrm{H} 12$ & 120.1 & $\mathrm{C} 24-\mathrm{C} 25-\mathrm{H} 25$ & 119.9 \\
\hline $\mathrm{C} 11-\mathrm{C} 12-\mathrm{H} 12$ & 120.1 & $\mathrm{C} 26-\mathrm{C} 25-\mathrm{H} 25$ & 119.9 \\
\hline $\mathrm{C} 12-\mathrm{C} 13-\mathrm{C} 14$ & $120.3(2)$ & $\mathrm{C} 25-\mathrm{C} 26-\mathrm{C} 21$ & $120.2(2)$ \\
\hline $\mathrm{C} 12-\mathrm{C} 13-\mathrm{H} 13$ & 119.9 & $\mathrm{C} 25-\mathrm{C} 26-\mathrm{H} 26$ & 119.9 \\
\hline $\mathrm{C} 14-\mathrm{C} 13-\mathrm{H} 13$ & 119.9 & $\mathrm{C} 21-\mathrm{C} 26-\mathrm{H} 26$ & 119.9 \\
\hline $\mathrm{C} 15-\mathrm{C} 14-\mathrm{C} 13$ & $120.4(2)$ & $\mathrm{C} 2-\mathrm{N} 1-\mathrm{N} 2$ & $110.84(16)$ \\
\hline $\mathrm{C} 15-\mathrm{C} 14-\mathrm{H} 14$ & 119.8 & $\mathrm{C} 3-\mathrm{N} 2-\mathrm{N} 1$ & $111.29(17)$ \\
\hline $\mathrm{C} 13-\mathrm{C} 14-\mathrm{H} 14$ & 119.8 & $\mathrm{~N} 2-\mathrm{C} 3-\mathrm{C} 31$ & $121.5(2)$ \\
\hline $\mathrm{C} 14-\mathrm{C} 15-\mathrm{C} 16$ & $119.8(3)$ & $\mathrm{N} 2-\mathrm{C} 3-\mathrm{H} 3$ & 119.2 \\
\hline $\mathrm{C} 14-\mathrm{C} 15-\mathrm{H} 15$ & 120.1 & $\mathrm{C} 31-\mathrm{C} 3-\mathrm{H} 3$ & 119.2 \\
\hline $\mathrm{C} 16-\mathrm{C} 15-\mathrm{H} 15$ & 120.1 & $\mathrm{C} 32-\mathrm{C} 31-\mathrm{C} 36$ & $119.1(2)$ \\
\hline $\mathrm{C} 15-\mathrm{C} 16-\mathrm{C} 11$ & $120.2(2)$ & $\mathrm{C} 32-\mathrm{C} 31-\mathrm{C} 3$ & $119.3(2)$ \\
\hline $\mathrm{C} 15-\mathrm{C} 16-\mathrm{H} 16$ & 119.9 & $\mathrm{C} 36-\mathrm{C} 31-\mathrm{C} 3$ & $121.6(2)$ \\
\hline $\mathrm{C} 11-\mathrm{C} 16-\mathrm{H} 16$ & 119.9 & $\mathrm{C} 31-\mathrm{C} 32-\mathrm{C} 33$ & $120.4(2)$ \\
\hline $\mathrm{O} 1-\mathrm{C} 1-\mathrm{C} 11$ & $122.96(19)$ & $\mathrm{C} 31-\mathrm{C} 32-\mathrm{H} 32$ & 119.8 \\
\hline $\mathrm{O} 1-\mathrm{C} 1-\mathrm{C} 2$ & $117.85(19)$ & $\mathrm{C} 33-\mathrm{C} 32-\mathrm{H} 32$ & 119.8 \\
\hline $\mathrm{C} 11-\mathrm{C} 1-\mathrm{C} 2$ & $119.19(19)$ & $\mathrm{C} 34-\mathrm{C} 33-\mathrm{C} 32$ & $120.2(2)$ \\
\hline $\mathrm{N} 1-\mathrm{C} 2-\mathrm{C} 21$ & $120.24(19)$ & $\mathrm{C} 34-\mathrm{C} 33-\mathrm{H} 33$ & 119.9 \\
\hline $\mathrm{N} 1-\mathrm{C} 2-\mathrm{C} 1$ & $120.61(18)$ & $\mathrm{C} 32-\mathrm{C} 33-\mathrm{H} 33$ & 119.9 \\
\hline $\mathrm{C} 21-\mathrm{C} 2-\mathrm{C} 1$ & $118.91(18)$ & $\mathrm{C} 33-\mathrm{C} 34-\mathrm{C} 35$ & $120.1(2)$ \\
\hline $\mathrm{C} 22-\mathrm{C} 21-\mathrm{C} 26$ & 118.99 (19) & $\mathrm{C} 33-\mathrm{C} 34-\mathrm{H} 34$ & 120.0 \\
\hline $\mathrm{C} 22-\mathrm{C} 21-\mathrm{C} 2$ & $120.9(2)$ & $\mathrm{C} 35-\mathrm{C} 34-\mathrm{H} 34$ & 120.0 \\
\hline $\mathrm{C} 26-\mathrm{C} 21-\mathrm{C} 2$ & $120.1(2)$ & $\mathrm{C} 36-\mathrm{C} 35-\mathrm{C} 34$ & $119.9(2)$ \\
\hline $\mathrm{C} 23-\mathrm{C} 22-\mathrm{C} 21$ & $120.4(2)$ & $\mathrm{C} 36-\mathrm{C} 35-\mathrm{H} 35$ & 120.0 \\
\hline $\mathrm{C} 23-\mathrm{C} 22-\mathrm{H} 22$ & 119.8 & $\mathrm{C} 34-\mathrm{C} 35-\mathrm{H} 35$ & 120.0 \\
\hline $\mathrm{C} 21-\mathrm{C} 22-\mathrm{H} 22$ & 119.8 & $\mathrm{C} 35-\mathrm{C} 36-\mathrm{C} 31$ & $120.3(2)$ \\
\hline $\mathrm{C} 24-\mathrm{C} 23-\mathrm{C} 22$ & $120.0(2)$ & $\mathrm{C} 35-\mathrm{C} 36-\mathrm{H} 36$ & 119.8 \\
\hline $\mathrm{C} 24-\mathrm{C} 23-\mathrm{H} 23$ & 120.0 & $\mathrm{C} 31-\mathrm{C} 36-\mathrm{H} 36$ & 119.8 \\
\hline $\mathrm{C} 22-\mathrm{C} 23-\mathrm{H} 23$ & 120.0 & & \\
\hline $\mathrm{C} 16-\mathrm{C} 11-\mathrm{C} 12-\mathrm{C} 13$ & $0.7(3)$ & $\mathrm{C} 2-\mathrm{C} 21-\mathrm{C} 22-\mathrm{C} 23$ & $-178.2(2)$ \\
\hline $\mathrm{C} 1-\mathrm{C} 11-\mathrm{C} 12-\mathrm{C} 13$ & $179.7(2)$ & $\mathrm{C} 21-\mathrm{C} 22-\mathrm{C} 23-\mathrm{C} 24$ & $-0.4(4)$ \\
\hline $\mathrm{C} 11-\mathrm{C} 12-\mathrm{C} 13-\mathrm{C} 14$ & $0.2(4)$ & $\mathrm{C} 22-\mathrm{C} 23-\mathrm{C} 24-\mathrm{C} 25$ & $-0.3(4)$ \\
\hline $\mathrm{C} 12-\mathrm{C} 13-\mathrm{C} 14-\mathrm{C} 15$ & $-1.0(4)$ & $\mathrm{C} 23-\mathrm{C} 24-\mathrm{C} 25-\mathrm{C} 26$ & $0.8(4)$ \\
\hline $\mathrm{C} 13-\mathrm{C} 14-\mathrm{C} 15-\mathrm{C} 16$ & $0.7(5)$ & $\mathrm{C} 24-\mathrm{C} 25-\mathrm{C} 26-\mathrm{C} 21$ & $-0.5(4)$ \\
\hline $\mathrm{C} 14-\mathrm{C} 15-\mathrm{C} 16-\mathrm{C} 11$ & $0.2(4)$ & $\mathrm{C} 22-\mathrm{C} 21-\mathrm{C} 26-\mathrm{C} 25$ & $-0.2(4)$ \\
\hline $\mathrm{C} 12-\mathrm{C} 11-\mathrm{C} 16-\mathrm{C} 15$ & $-1.0(3)$ & $\mathrm{C} 2-\mathrm{C} 21-\mathrm{C} 26-\mathrm{C} 25$ & $178.7(2)$ \\
\hline $\mathrm{C} 1-\mathrm{C} 11-\mathrm{C} 16-\mathrm{C} 15$ & $-179.9(2)$ & $\mathrm{C} 21-\mathrm{C} 2-\mathrm{N} 1-\mathrm{N} 2$ & $-178.27(18)$ \\
\hline
\end{tabular}




\begin{tabular}{llll}
$\mathrm{C} 16-\mathrm{C} 11-\mathrm{C} 1-\mathrm{O} 1$ & $178.1(2)$ & $\mathrm{C} 1-\mathrm{C} 2-\mathrm{N} 1-\mathrm{N} 2$ & $-3.8(3)$ \\
$\mathrm{C} 12-\mathrm{C} 11-\mathrm{C} 1-\mathrm{O} 1$ & $-0.8(3)$ & $\mathrm{C} 2-\mathrm{N} 1-\mathrm{N} 2-\mathrm{C} 3$ & $179.9(2)$ \\
$\mathrm{C} 16-\mathrm{C} 11-\mathrm{C} 1-\mathrm{C} 2$ & $-2.4(3)$ & $\mathrm{N} 1-\mathrm{N} 2-\mathrm{C} 3-\mathrm{C} 31$ & $176.64(19)$ \\
$\mathrm{C} 12-\mathrm{C} 11-\mathrm{C} 1-\mathrm{C} 2$ & $178.7(2)$ & $\mathrm{N} 2-\mathrm{C} 3-\mathrm{C} 31-\mathrm{C} 32$ & $-180.0(2)$ \\
$\mathrm{O} 1-\mathrm{C} 1-\mathrm{C} 2-\mathrm{N} 1$ & $-100.0(2)$ & $\mathrm{N} 2-\mathrm{C} 3-\mathrm{C} 31-\mathrm{C} 36$ & $-1.3(3)$ \\
$\mathrm{C} 11-\mathrm{C} 1-\mathrm{C} 2-\mathrm{N} 1$ & $80.6(3)$ & $\mathrm{C} 36-\mathrm{C} 31-\mathrm{C} 32-\mathrm{C} 33$ & $-1.4(4)$ \\
$\mathrm{O} 1-\mathrm{C} 1-\mathrm{C} 2-\mathrm{C} 21$ & $74.6(3)$ & $\mathrm{C} 3-\mathrm{C} 31-\mathrm{C} 32-\mathrm{C} 33$ & $177.3(2)$ \\
$\mathrm{C} 11-\mathrm{C} 1-\mathrm{C} 2-\mathrm{C} 21$ & $-104.9(2)$ & $\mathrm{C} 31-\mathrm{C} 32-\mathrm{C} 33-\mathrm{C} 34$ & $1.5(4)$ \\
$\mathrm{N} 1-\mathrm{C} 2-\mathrm{C} 21-\mathrm{C} 22$ & $-176.3(2)$ & $\mathrm{C} 32-\mathrm{C} 33-\mathrm{C} 34-\mathrm{C} 35$ & $-0.4(4)$ \\
$\mathrm{C} 1-\mathrm{C} 2-\mathrm{C} 21-\mathrm{C} 22$ & $9.1(3)$ & $\mathrm{C} 33-\mathrm{C} 34-\mathrm{C} 35-\mathrm{C} 36$ & $-0.7(4)$ \\
$\mathrm{N} 1-\mathrm{C} 2-\mathrm{C} 21-\mathrm{C} 26$ & $4.9(3)$ & $\mathrm{C} 34-\mathrm{C} 35-\mathrm{C} 36-\mathrm{C} 31$ & $0.7(4)$ \\
$\mathrm{C} 1-\mathrm{C} 2-\mathrm{C} 21-\mathrm{C} 26$ & $-169.7(2)$ & $\mathrm{C} 32-\mathrm{C} 31-\mathrm{C} 36-\mathrm{C} 35$ & $0.4(4)$ \\
$\mathrm{C} 26-\mathrm{C} 21-\mathrm{C} 22-\mathrm{C} 23$ & $0.6(4)$ & $\mathrm{C} 3-\mathrm{C} 31-\mathrm{C} 36-\mathrm{C} 35$ & $-178.3(2)$ \\
\hline
\end{tabular}

Hydrogen-bond geometry $\left(\AA,{ }^{\circ}\right)$

$\mathrm{Cg}$ is the centroid of the $\mathrm{C} 31-\mathrm{C} 36$ phenyl ring.

\begin{tabular}{lllll}
\hline$D-\mathrm{H} \cdots A$ & $D-\mathrm{H}$ & $\mathrm{H} \cdots A$ & $D \cdots A$ & $D-\mathrm{H} \cdots A$ \\
\hline $\mathrm{C} 35-\mathrm{H} 35 \cdots \mathrm{O} 1^{\mathrm{i}}$ & 0.95 & 2.61 & $3.337(3)$ & 134 \\
$\mathrm{C} 3-\mathrm{H} 3 \cdots \mathrm{O} 1^{\mathrm{ii}}$ & 0.95 & 2.41 & $3.272(3)$ & 151 \\
$\mathrm{C} 32-\mathrm{H} 32 \cdots \mathrm{O} 1^{\mathrm{ii}}$ & 0.95 & 2.68 & $3.478(3)$ & 141 \\
$\mathrm{C} 26-\mathrm{H} 26 \cdots \mathrm{Cg}^{\mathrm{iii}}$ & 0.95 & 2.97 & $3.699(3)$ & 135 \\
\hline
\end{tabular}

Symmetry codes: (i) $x,-y+1 / 2,-z+1 / 2$; (ii) $x+1 / 4, y-1 / 4,-z+3 / 4$; (iii) $x+1 / 4,-y+1 / 4, z+1 / 4$. 